
\title{
R Reseracth Sulure \\ Gene Homologies Identified between Trypanosoma cruzi Antigen 36 and Mammalian TRIM21 Genes Using Bioinformatics Analysis
}

Martin A Winkler ( $\square$ winklma01@gmail.com )

The Biotech Advisor

Alfred A Pan

TNTC, Inc.

\section{Research Article}

Keywords: Chagas' disease, Chronic Chagas Cardiomyopathy, Trypanosoma cruzi, Ro52, TRIM21, Antigen 36 (Ag 36), Needleman-Wunsch algorithm

Posted Date: June 2nd, 2021

DOI: https://doi.org/10.21203/rs.3.rs-553828/v1

License: (c) (1) This work is licensed under a Creative Commons Attribution 4.0 International License. Read Full License 


\section{Abstract}

Background: We previously reported that a Human Ro52 gene sequence (TRIM21) produced a significant stretch of protein sequence homologous to T. cruzi Antigen 36 (Ag 36) protein sequence, when Ag 36 was translated in the second reading frame. Comparison of their respective DNA sequences demonstrated a 114 nucleotide region of both genes having 70 percent partial homology. After Ro52 was shown to be an E3 Ubiquitin dependent Type I ligase for transcription factors for Interferon genes, we proposed that the Ag 36 gene, which contains a repetitive motif within it, may function to repress Ro52 in the human heart through RNA interference, or other unknown mechanism, giving rise to autoimmunity found in Chronic Chagas Cardiomyopathy (CCC).

Results: To test that hypothesis, we compared various mammalian TRIM genes to the T. cruzi Ag 36 DNA sequence using the Needleman-Wunsch algorithm in the http:Ilusegalaxy.eu bioinformatics tool base. In addition to human and chimpanzee, TRIM21 comparable gene regions from canine, shrew, ferret, bat, feline, and armadillo, and aardvark showed homology to the gene for Ag 36 ranging from 68 to 30 percent. However, mouse and eight other mammalian species showed no significant homology. Since mice have been shown to have severe cardiac cardiomyopathy after infection, but their TRIM21 was not homologous to Ag 36 in this study, we conclude that the gene homology has no causative link to CCC.

Conclusions: In addition to human TRIM21, eight mammalian species showed partial gene homology to T. cruzi Ag 36, and some of these have been demonstrated to have CCC. However, rats and mice TRIM21 showed no partial homology to Ag 36. Since these species have been demonstrated to have CCC, the partial gene homology between Ag36 and TRIM 21 may not be causative or associated with CCC, as was originally hypothesized.

\section{Background}

Chagas' disease has become a progressive emerging disease in the United States. In South and Central America, the disease may affect over 18 million people. Typically, the causative agent,

Trypanosomacruzi, may not immediately kill the host, but by its definition, the parasite keeps the host in check, in order to maximize transmission and therefore, prolong its life cycle. The mammalian host can gradually develop illness from an acute or mild form of the disease to a latent stage, to a chronic stage. There is significant mortality due to the Acute and Chronic stages of this disease ${ }^{1}$. In particular, Chronic Chagas Cardiomyopathy (CCC) is thought to be caused by an autoimmune attack upon nerve and/or heart tissue.

Recognition of this disease as a potential threat to the US blood supply was in 1992 when several biopharmaceutical companies developed diagnostic tests to identify this disease ${ }^{2}$. In 1995 the FDA accepted a 510(k) diagnostic test for Chagas' disease ${ }^{3,4}$. Progressive improvements to identify several highly antigenic $T$. cruzi molecules reactive with human chagasic antiserum were made in subsequent 
years (2006-2011); the American Association of Blood Banks (AABB), the FDA, CDC, and WHO have now recognized this disease as one of the 13 donor screening assays for Infectious Agents.

Presently there are no vaccines against Chagas' disease and therapeutic agents (benznidazole) have only recently (2017) been FDA approved for use in children ages 2 to 12 years old with Chagas' disease. Side effects are common, frequent, and severe with increasing age. In this regard, alternative methods for treatment are necessary. Our primary focus has been to search and identify highly antigenic $T$. cruzi molecules reactive with human chagasic antiserum ${ }^{2,3,5,6}$. To this end, one particular cloned gene from $T$. cruzi amastigotes was sequenced and found to be identical to the repetitive antigen Clone 36 ("Antigen $36 ")^{7}$.

Search of GenBank using our DNA sequence translated by the TFASTA program revealed a high degree of homology of Human Ro52 with the translated sequence in the second reading frame of Ag 36. Direct comparison of the Ag 36 DNA sequence with Ro52 DNA sequence revealed a significant homology of the Ag 36 DNA sequence to TRIM21, the gene for human Ro52 ${ }^{8}$. The TRIM21 (tripartite motif containing-21) gene protein product Ro52 was subsequently shown to be an E3 Ubiquitin ligase that modifies transcription factors for alpha and beta interferons and other cytokines ${ }^{9}$. Knock-out of Ro2 in mice strains showed that they were susceptible to tissue inflammation and systemic autoimmunity after injury induced by skin tagging ${ }^{10}$. However, the TRIM21 protein product Ro52 also can stimulate innate immunity, for example, in macrophages. There it acts in a non-degradation pathway of ubiquitination, as an E3 ligase for IRF-8 that upregulates cytokines such as IL-12/p40, and therefore contributes to innate immunity in macrophages ${ }^{11}$. TRIM21-deficient bone marrow-derived macrophages showed a reduced response to Toll-like receptor agonist Bacillus Calmette-Guerin ${ }^{12}$.

TRIM21 is also an antibody receptor and cytosolic ubiquitin ligase that provides a line of defense against invading viruses by acting as a sensor that intercepts antibody-coated viruses that have evaded extracellular neutralization and breached the cell membrane ${ }^{13-15}$. After interaction with the Fc of antibodies bound to the virus, the TRIM21 receptor triggers a coordinated effector and signaling response that prevents viral replication while at the same time inducing an anti-viral cellular state ${ }^{16}$. This dual effector function is tightly regulated by auto-ubiquitination and phosphorylation. In addition, TRIM21 has been studied extensively with antibody receptors bound to viruses but minimally with parasites ${ }^{16}$. We proposed that the partial gene homology between TRIM21 and Ag 36 may cause RNA interference, mRNA silencing, or other down-regulation of Ro52, leading to the autoimmunity of Chronic Chagas Cardiomyopathy (CCC ${ }^{17}$. We also suggested that the interference could be beneficial for the parasite, during its invasion of macrophages by blocking TRIM21 stimulation of innate immunity in these phagocytic cells. In this work, we extend the comparison of DNA sequences of Ag 36 to eighteen additional mammalian TRIM21 sequences, using online bioinformatics tools to determine significant partial homologies and to test whether the homology is associated with CCC.

\section{Results}




\section{Homology Comparison of TRIM21 (nucleotides 856-916) to Ag 36 in Mammals}

The human TRIM21 sequence in nucleotides region 856 to 916 had the greatest homology to $\mathrm{Ag} 36$ and was used for comparison. In the regions of Ag 36 gene homology compared to TRIM21 genes (TRIM21 base numbers 856 to 916$)$, the greatest percentage homology was human $(68 \%)$, followed by chimpanzee (60\%), dog (57\%) and shrew (50\%). Seven other species showed partial homologies in the compared region of 43 to 17\% (Table 1). The nine other mammalian TRIM21 genes shown in the Table 1 had no significant homology in this region. 
Table 1 Percent Homology Comparison of TRIM21

(856-916) in Mammals using the Needleman-

Wunsch Alignment of T. cruzi Ag 36

\begin{tabular}{|c|c|c|c|}
\hline Mammal & Genus/Species & $\begin{array}{l}\text { \# } \\
\text { Nucleotides } \\
\text { Homologous } \\
\text { in TRIM21 } \\
856-916\end{array}$ & $\begin{array}{l}\text { Percent } \\
\text { Homology }\end{array}$ \\
\hline Human & Homo sapiens & $41 / 60$ & $68 \%$ \\
\hline Chimpanzee & $\begin{array}{l}\text { Pan } \\
\text { Trogolodytus }\end{array}$ & $36 / 60$ & $60 \%$ \\
\hline Dog & $\begin{array}{l}\text { Canis } \\
\text { familiiaris }\end{array}$ & $34 / 60$ & $57 \%$ \\
\hline Shrew & Sorex araneus & $30 / 60$ & $50 \%$ \\
\hline Ferret & $\begin{array}{l}\text { Mustela } \\
\text { potorius furo }\end{array}$ & $27 / 60$ & $43 \%$ \\
\hline Bat, common Vampire & $\begin{array}{l}\text { Desmodus } \\
\text { rotundus }\end{array}$ & $26 / 60$ & $43 \%$ \\
\hline Cat, domestic & Felis cattus & $24 / 60$ & $40 \%$ \\
\hline Armadillo (9 band) & $\begin{array}{l}\text { Dasypus } \\
\text { ovemcinctus }\end{array}$ & $23 / 60$ & $38 \%$ \\
\hline Deer, White tailed & $\begin{array}{l}\text { Otocoileus } \\
\text { virginiansus }\end{array}$ & $19 / 60$ & $32 \%$ \\
\hline Aardvark & $\begin{array}{l}\text { Orycteropus } \\
\text { afer }\end{array}$ & $20 / 60$ & $30 \%$ \\
\hline Rabbit & $\begin{array}{l}\text { Oryctuolagus } \\
\text { cuniculus }\end{array}$ & $19 / 60$ & $17 \%$ \\
\hline Rat & $\begin{array}{l}\text { Rattus } \\
\text { norwegicus }\end{array}$ & $0 / 60$ & $0 \%$ \\
\hline Mouse & Mus musculus & $0 / 60$ & $0 \%$ \\
\hline Gerbil & $\begin{array}{l}\text { Meriones } \\
\text { unguaiculatus }\end{array}$ & $0 / 60$ & $0 \%$ \\
\hline Sheep & Ovis aires & $0 / 60$ & $0 \%$ \\
\hline Cow & Bos taurus & $0 / 60$ & $0 \%$ \\
\hline Horse & $\begin{array}{l}\text { Equus } \\
\text { caballus }\end{array}$ & $0 / 60$ & $0 \%$ \\
\hline Goat & Capra hircus & $0 / 60$ & $0 \%$ \\
\hline Marmoset & Callithex & $0 / 60$ & $0 \%$ \\
\hline
\end{tabular}


The mammalian TRIM21 genes were compared to T. cruzi Ag 36 by the Needleman-Wunsch Alignment at www.usegalaxy.org (please see Methods). The number of nucleotides that were homologous in the TRIM21 nucleotide 856 to 916 range were counted and divided by 60 , the total nucleotides in this range.

\section{Discussion}

Human TRIM21 gene sequence was shown to have a significant stretch of DNA sequence homologous to T. cruzi Ag 36. Comparison of Ag 36 to mammalian TRIM21 DNA sequences demonstrated a 114 nucleotide region ranging from 68 to $38 \%$ partial homology in human, chimpanzee, canine, armadillo, and ten other species (Table 1). Of these species, humans and dogs have been shown to have CCC after $T$. cruzi infection ${ }^{18-21}$. Domesticated cats have been known to manifest the acute stages of Chagas' disease and there is only limited data of CCC in these animals ${ }^{22}$. Ro52 is an E3 Ubiquitin dependent Type I ligase needed for transcription factors for Interferon genes. It was proposed that the Ag 36 sequence motif, repeated at least three times ${ }^{7}$ would increase the chances of the homology's putative effect ${ }^{17}$. If this gene homology has an outcome on the infected host, for example, through RNA interference or mRNA silencing, decreased expression of Ro52 may result, giving rise to the autoimmunity found in CCC, and conferring an advantage for the parasite in infecting monocytes. However, mice have been shown to have severe cardiac cardiomyopathy after $T$. cruzi infection ${ }^{23}$. Our investigations showed no homology of mouse TRIM21 to Ag 36, implying that there may be no causative or associative link to CCC from the homology of TRIM21 to Ag 36. There is only one isoform and no splice variants of TRIM21 reported in laboratory mice, which are inbred strains. The TRIM 21 gene therefore does not show variability in laboratory mice, ruling out the possibility of the homology being in them. It would be of interest to determine if TRIM21 in wild mice shows splice variants or isoforms, and if so, whether they display CCC when infected with $T$. cruzi. TRIM genes are a large family of mammalian and vertebrate genes, which share homologous domains ${ }^{14,24}$. Consequently, since TRIM21 is homologous to many genes in this family, it would be worthwhile to examine mammalian TRIM genes for homology to Ag 36 as well. The results demonstrate the power of a comparative bioinformatics analysis of these TRIM 21 genes between the mammalian species.

\section{Conclusions}

In this study, we used a comparative bioinformatics approach to determine whether the partial homology observed of Human TRIM21 to T. cruzi Ag 36 was observed in 19 other mammalian species, and if observed homologies correlated with CCC in those species. Ten species demonstrated the partial homology by the Needleman-Wunsch Algorithm. Nine species demonstrated no homology. However, rats 
and mice lacked the homology, though they have been shown to have CCC. This exception rules out the partial homology as a possible cause or association with CCC.

\section{Methods}

Sequences of TRIM21s from 19 mammalian species were retrieved from Genbank or the University of California Santa Barbara databases, and sequences compared to the Ag 36 gene (Genbank M21331) with tools at https://usegalaxy.org ${ }^{25,26}$. The mammalian TRIM21 analyzed were: primates (human, chimpanzee), mouse, rat, gerbil, dog, cat, nine-banded armadillo, shrew, lynx, ferret, marmoset, bat, sheep, cow, deer, horse, and goat. Gene sequences were aligned by the Needleman-Wunsch algorithm ${ }^{27}$. The Needleman-Wunsch global alignment tool is an algorithm used to find the optimum alignment (including gaps) of two sequences when considering their entire length. The workflow and results are stored at the URL https://usegalaxy.eu/u/martinawinklerphd/h/copy-of-copy-of-mammalian-trim-genes-comparedwith-antigen-36. The bioinformatics workflow is visible to all, but registration (which is free) is required to view the results.

\section{Declarations}

\section{Supplementary Information}

The online version contains supplementary material available at https://

\section{Acknowledgements}

The authors would like to thank Abbott Laboratories and our colleagues where the foundational investigations were performed.

\section{Authors' contributions}

MAW and AAP conceptualized the research questions. MAW did the computational data analysis. MAW and AAP and wrote the manuscript, reviewed and supervised the work. All authors read and approved the final manuscript.

\section{Funding}

The Galaxy server that was used for some calculations is in part funded by Collaborative Research Centre 992 Medical Epigenetics (DFG grant SFB 992/1 2012) and German Federal Ministry of Education and Research (BMBF grants 031 A538A/A538C RBC, 031L0101B/031L0101C de.NBI-epi, 031L0106 de.STAIR $($ de.NBI)).

\section{Availability of data and materials}


The workflow and results are stored at the URL: https://usegalaxy.eu/u/martinawinklerphd/h/copy-ofcopy-of-mammalian-trim-genes-compared-with-antigen-36. The bioinformatics workflow is visible to all, but registration (which is free) is required to view the results.

\section{Ethics approval and consent to participate}

Not applicable.

\section{Consent for publication}

Not applicable.

\section{Competing interests}

The author(s) declared no potential conflicts of interest with respect to the research, authorship, and/or publication of this article.

\section{Author details}

${ }^{1}$ The Biotech Advisor, Lawrence, KS, USA. ${ }^{2}$ TNTC, Inc., Pleasant Hill, CA, USA.

\section{Abbreviations}

Ag: Antigen; CCC: Chronic Chagas Cardiomyopathy; TRIM21: Tripartite Motif containing-21

\section{References}

1. Aiello VD, de Campos FPF. Chronic Chagas cardiomyopathy. Autopsy Case Rep 2015;5(3):7-9. doi: 10.4322/acr.2015.012.

2. Pan AA, Rosenberg GB, Hurley MK, Schock GJH, Chu VP, Aiyappa AA. Clinical evaluation of an EIA for the sensitive and specific detection of serum antibody to Trypanosomacruzi (Chagas' disease). J Infect Dis 1992;165(3):585-588. doi: 10.1093/infdis/165.3.585.

3. Brashear RJ, Winkler MA, Schur JD, Lee H, Burczak JD, Hall HJ, Pan AA. Detection of antibodies to Trypanosomacruzi among blood donors in the southwestern and western United States. I. Evaluation of the sensitivity and specificity of an enzyme immunoassay for detecting antibodies to $T$. cruzi. Transfusion 1995;35(3):213-218. doi: 10.1046/j.1537-2995.1995.35395184277.x.

4. Pan AA, Winkler MA. The threat of Chagas' disease in transfusion medicine. The presence of antibodies to Trypanosomacruzi in the US blood supply. Lab Med 1995;28:269-274.

5. Pan AA, McMahon-Pratt D. Amastigote and epimastigote stage-specific components of Trypanosomacruzi characterized by using monoclonal antibodies. Purification and molecular characterization of an 83-kilodalton amastigote protein. J Immunol 1989;143(3):1001-1008. PMID: 2501384. 
6. Winkler MA, Brashear RJ, Hall HJ, Schur JD, Pan AA. Detection of antibodies to Trypanosomacruzi among blood donors in the southwestern and western United States. II. Evaluation of a supplemental enzyme immunoassay and radioimmunoprecipitation assay for confirmation of seroreactivity. Transfusion 1995;35(3):219-225. doi: 10.1046/j.1537-2995.1995.35395184278.x

7. Ibañez CF, Affranchino JL, Macina RA, Reyes MB, Leguizamon S, Camargo ME, Lena Åslund L, Pettersson U, Frasch, ACC. Multiple Trypanosomacruzi antigens containing tandemly repeated amino acid sequence motifs. Mol Biochem Parasitol 1988;30(1):27-34. doi: 10.1016/0166-6851(88)901296.

8. Winkler MA, Rivera DM, Pan AA, Nowlan SF. Homology of Trypanosomacruzi clone 36 repetitive DNA sequence to sequence encoding human Ro/SSA 52 kD autoantigen. Parasite 1998;5(1):94-95. PMID: 9754305..

9. Higgs R, Gabhann JN, Larbi NB, Breen EP, Fitzgerald KA, Jefferies C. The E3 ubiquitin ligase Ro52 negatively regulates IFN- production post-pathogen recognition by polyubuiquitin-mediated degradation of IRF3. J Immunol 2008;181(3):1780-1786. doi: 10.4049/jimmunol.181.3.1780.

10. Espinosa A, Dardalho, V, Brauner, Ambrosi A, Higgs R, Quintana FJ, Sjostrand M, Eloranta M-L, Gabhann, JN, Winqvist O, Sundelin, B, Jefferies CA, Rozell B, Kuchroo, VK, Wahren-Herlenius, M. Loss of the lupus autoantigen Ro52/Trim21 induces tissue inflammation and systemic autoimmunity by disregulating the IL-23-Th17 pathway. J Exp Med 2009;206(8):1661-1671. doi: 10.1084/jem.20090585.

11. Kong HJ, Anderson DE, Lee CH, Jang MK, Tamura T, Tailor P, Cho HK, Cheong JH, Xiong H, Morse III, $\mathrm{HC}$, et al. Autoantigen Ro52 is an interferon inducible E3 ligase that ubiquitinates IRF-8 and enhances cytokine expression in macrophages. J Immunol 2007;179(1):26-30. doi: 10.4049/jimmunol.179.1.26.

12. Sjostrand M, Carrow B, Espinosa A. TRIM21 controls toll-like receptor 2 responses in bone-marrowderived macrophages. Immunology 2020;159(3):335-343. doi: 10.1111/imm.13157.

13. Meroni G, Diez-Roux G. TRIM/RBCC, a novel class of single protein RING finger' E3 ubiquitin ligases. Bioessays 2005;27(11):1147-1157. doi: 10.1002/bies.20304.

14. Ozato $\mathrm{K}$, Shin DM, Chang TH, Morse HC III. TRIM family proteins and their emerging roles in innate immunity. Nat Rev Immunol. 2008(11);8:849-860. doi: 10.1038/nri2413.

15. Nisole S, Stoye JP, Saib A. TRIM family proteins: retroviral restriction and antiviral defence. Nat Rev Microbiol 2005;3(10):799-808. doi: 10.1038/nrmicro1248.

16. Burbelo PD, Teos LY, Herche JL, ladarola MJ, Aleviizos II. Autoantigens against the immunoglobulinbinding region of Ro2 link its autoantigenicity with pathogen neutralization. Sci Rep 2018;8(1):1-11. doi: 10.1038/s41598-018-21522-7.

17. Winkler MA, Pan AA. Is there a link between the human TRIM21 and Trypanosomacruzi clone 36 genes in Chagas' disease? Mol Immunol 2010;48(1-3):365-367. doi: 10.1016/j.molimm.2010.07.013.

18. Andrade Z. The canine model of Chagas' Disease. Mem Inst Oswaldo Cruz, Rio de Janeiro, Suppl. 1984;79:77-83. 
19. Gutierrez FRS, Guedes PMM, Gazzinelli RT, Silva JS. The role of parasite persistence in pathogenesis of Chagas heart disease. Parasite Immunol 2009;31(11):673-685. doi: 10.1111/j.13653024.2009.01108.x

20. Santos FM, Mazzeti AL, Caldas S, Concalves KR, Lime WG, Torres RM, Bahia MT. Chagas cardiomyopoathy: The potential effect of benznidazole treatment on diastolic dysfunction and cardiac damage in dogs chronically infected with Trypanosomacruzi. Acta Tropica 2016;161:44-54. doi: 10.1016/j.actatropica.2016.05.007.

21. Jansen AM, Xavier SCC, Roque ALR. 11-Ecological aspects of Trypanosoma cruzi: Wild hosts and reservoirs. American Trypanosomiasis Chagas' Disease (Second Edition), One Hundred Years of Research 2017;243-264.

22. Jimenez-Coell M, Acosta-Viana KY, Guzman-Marin E, Gomez-Rios A, Ortega-Pacheco A. Epidemiological survey of Trypanosoma cruzi infection in domestic owned cats from the tropical southeast of Mexico. Zoonoses Public Health 2012;59(Suppl 2):102-109. doi: 10.1111/j.18632378.2012.01463.x

23. Hoffman KA, Reynolds C, Bottazzi ME, Hotez P, Jones K. Improved biomarker and imaging analysis for characterizing progressive cardiac fibrosis in a mouse model of chronic Chagasic cardiomyopathy. J Am Heart Assoc 2019;8(22):1-15. doi: 10.1161/JAHA.119.013365.

24. Sardiello M, Cairo S, Fontanella B, Ballabio A, Meroni G. Genomic analysis of the TRIM family reveals two groups of genes with distinct evolutionary properties. BMC Evol Biol 2008,8:225-247. doi:10.1186/1471-2148-8-225

25. Afgan E, Baker D, van den Beek M, Blankenberg D, Bouvier D, Cech M, Chilton J, Clements D, Coraor N, Eberhard C, et al. The Galaxy platform for accessible, reproducible and collaborative biomedical analyses. Nucl Acid Res 2016;44(W1):W3-W10. doi:10.1093/nar/gkw343.

26. Blankenberg D, Taylor J, Schenck I, He J, Zhang Y, Ghent M, Veeraaghavan N, Albert I, Miller W, Makova KD, Hardison RC, Nekrutenko A. A framework for collaborative analysis of ENCODE data: Making large-scale analyses biologist-friendly. Genome Res 2007;17(6):960-964. doi.org/10.1101/gr.5578007.

27. Rice P, Longden I, Bleasby A. EMBOSS: The European Molecular Biology Open Software Suite. Trends in Genet 2000;16(6):276-277. doi.org/10.1016/S0168-9525(00)02024-2.

\section{Figures}


M34551.1

889

M21331.1

42

M34551.1

928

M21331.1

88

M34551.1

977

M21331.1

114
851 TGTGTGCCATGTGCCAGG----GCTGAAGAAGATGCTGAGG-------AC

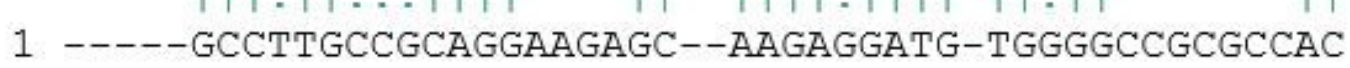

890 ATGTG---CAGTCCACATCACTCTGGA----TCCAGAC----ACAGCCAA

$$
.|1| \quad|.|-|| 1|.||| .|1 .| 1 \quad|1 .| 1|| \quad|1| 1.1
$$

43 GT-TGATCCCGACCACTTC-CGCTCGACGACTCAAGACGCGTACAGGC--

929 TCCGTGGCTGATACTTTCAGAAGATCGGAG-ACAAGTGAGGCTTGGAGAC

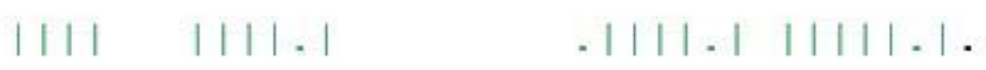

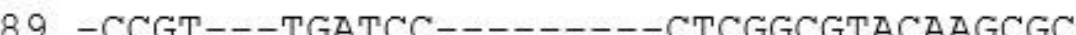

CTCGGCGTACAAGCGC----------

\section{Figure 1}

Comparison of TRIM21 from human with T. cruzi Ag 36, using the Needleman-Wunsch Algorithm, which is a global alignment algorithm to find the optimum alignment (including gaps) of two sequences when considering their entire length.

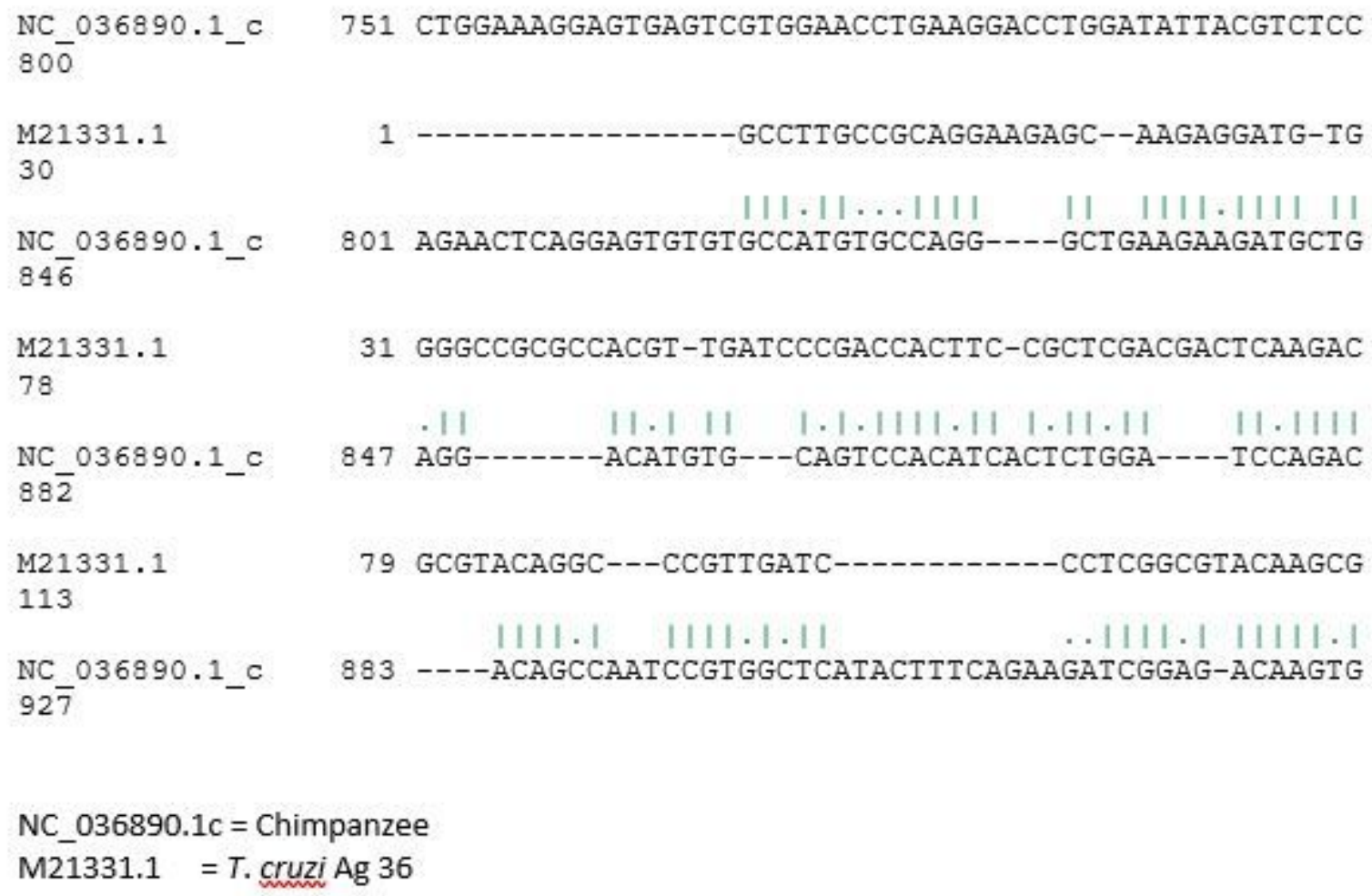




\section{Figure 2}

Comparison of TRIM21 from chimpanzee with T. cruzi Ag 36, using the Needleman-Wunsch Algorithm, which is a global alignment algorithm to find the optimum alignment (including gaps) of two sequences when considering their entire length.

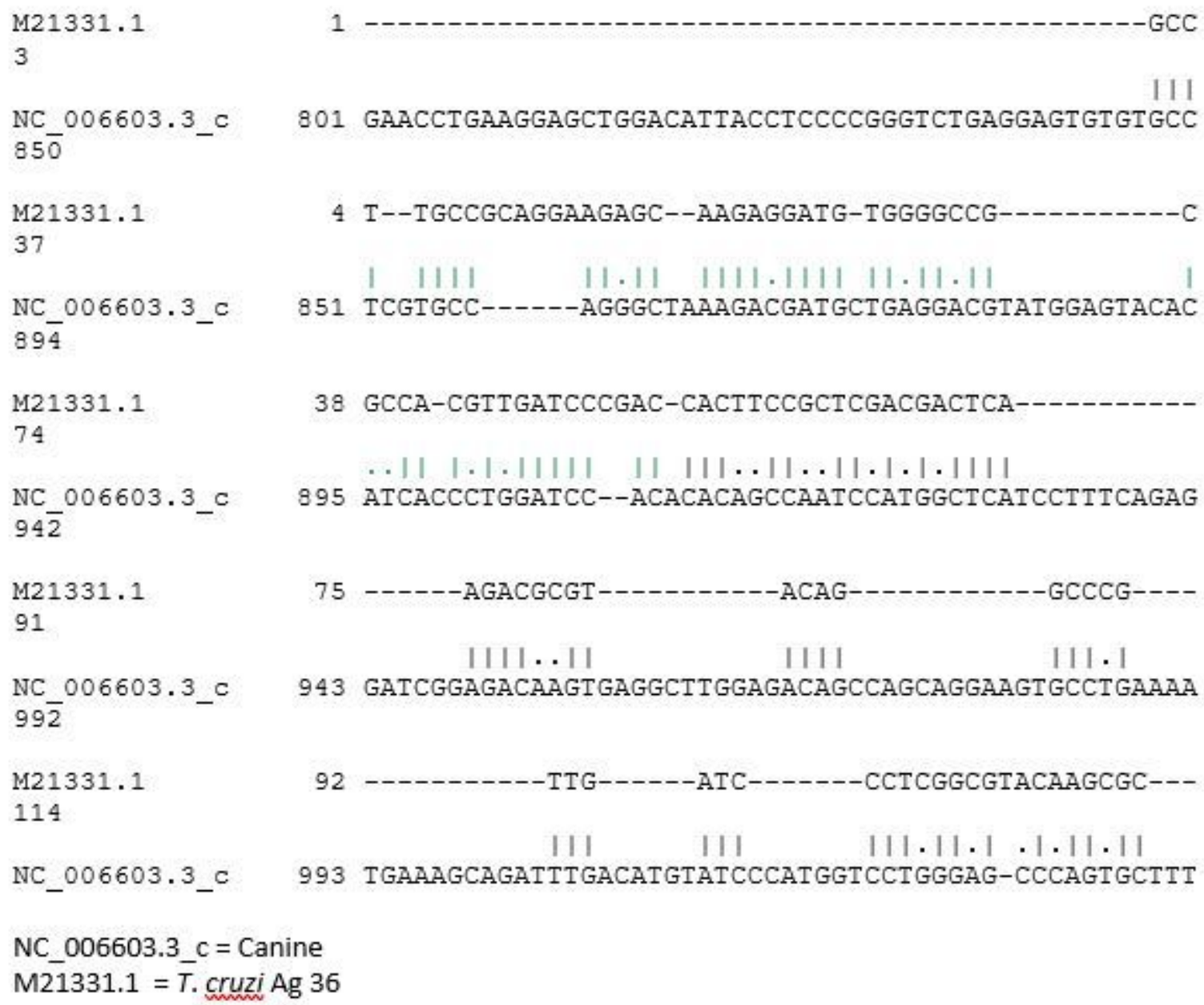

\section{Figure 3}

Comparison of TRIM21 from dog with T. cruzi Ag 36, using the Needleman-Wunsch Algorithm, which is a global alignment algorithm to find the optimum alignment (including gaps) of two sequences when considering their entire length. 


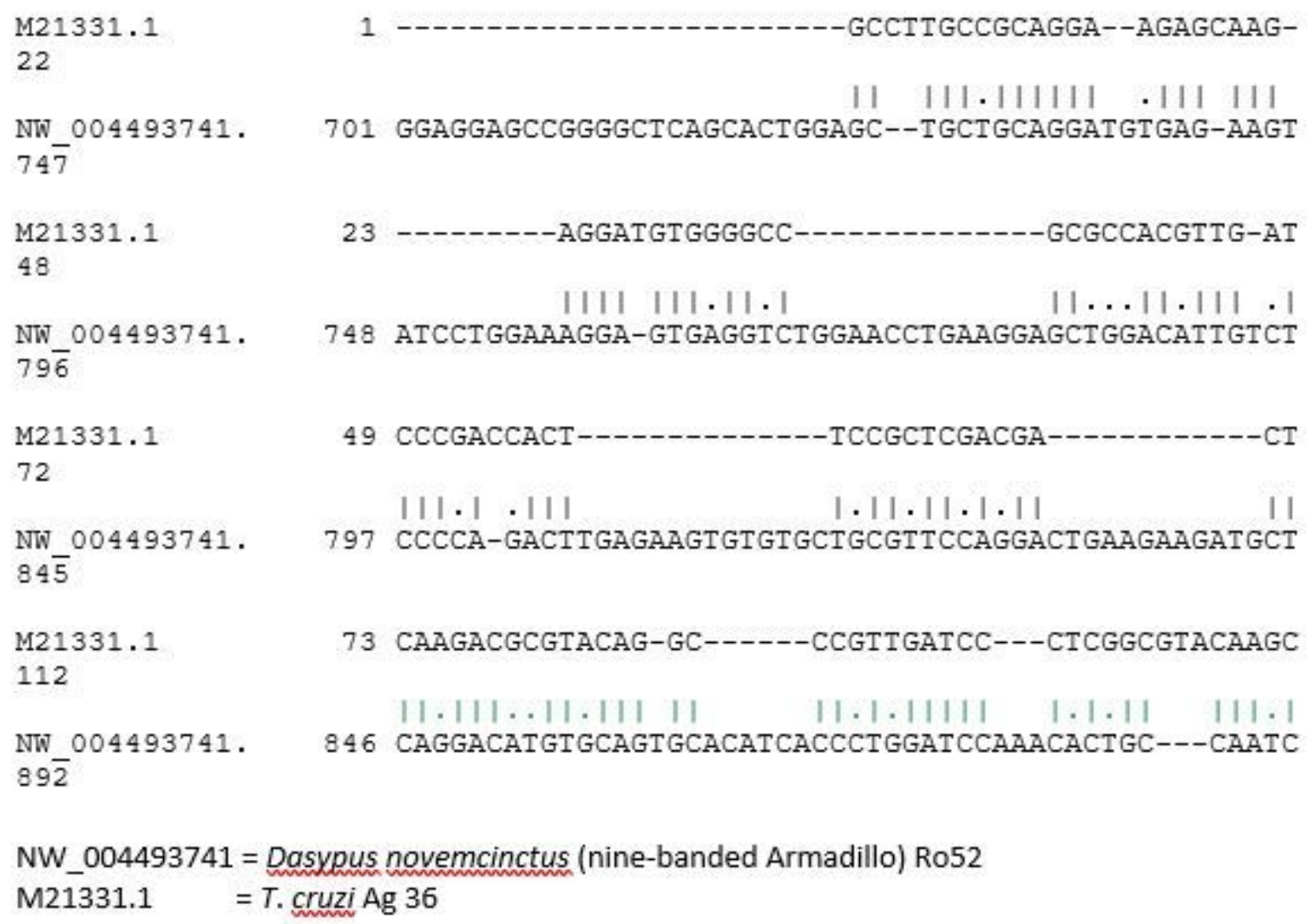

Figure 4

Comparison of TRIM21 from Nine-banded Armadillo with T. cruzi Ag 36, using the Needleman-Wunsch Algorithm, which is a global alignment algorithm to find the optimum alignment (including gaps) of two sequences when considering their entire length. 